\title{
Comparison of the Development Diabetic Induced Renal Disease in Strains of Goto-Kakizaki Rats
}

\author{
Naoki Kojima ${ }^{1,2}$, Tiffani N. Slaughter ${ }^{1}$, Adrienne Paige ${ }^{1}$, Sota Kato ${ }^{1,2}$, Richard J. Roman ${ }^{1}$ and Jan M. Williams ${ }^{1 *}$ \\ ${ }^{1}$ Department of Pharmacology and Toxicology, University of Mississippi Medical Center, USA \\ ${ }^{2}$ Molecular Function and Pharmacology Laboratories, Taisho Pharmaceutical Co., Ltd., Japan
}

\begin{abstract}
This study compared temporal changes in renal hemodynamics, proteinuria and the development of renal disease in Goto-Kakizaki (GK) type II diabetic rats that are resistant to the development of diabetic nephropathy and a genetically modified GK substrain (T2DN) carrying the mitochondrial genome and other alleles from Fawn hooded-hypertensive $(\mathrm{FHH})$ rats is more susceptible to the development of renal injury. Both GK and T2DN rats were diabetic (>250 $\mathrm{mg} / \mathrm{dL}$ ) and blood glucose levels were not significantly different at 3,6 and 18 months of age. Blood pressure was also similar in both strains at all 3 ages. Renal blood flow (RBF) was $45 \%$ higher in 3 month old T2DN rats than GK rats but glomerular filtration rate (GFR) was similar. T2DN rats exhibited a progressive increase in proteinuria from $41 \pm 2$ to $524 \pm 50 \mathrm{mg} /$ day and $57 \%$ fall in GFR as they aged from 3 to 18 months of age. In contrast, proteinuria only increased to $162 \pm 31 \mathrm{mg} /$ day in GK and GFR remained unaltered. The kidneys from 18 month old T2DN rats exhibited severe glomerulosclerosis, interstitial fibrosis and tubular necrosis while kidneys from GK rats did not. Plasma creatinine levels were 2.4 fold higher in 18 month old T2DN than in GK rats. These data demonstrate that T2DN rats develop most of the features of diabetic nephropathy including progressive proteinuria and chronic kidney disease whereas the closely related GK strain does not, even though blood pressure and the level of hyperglycemia are similar.
\end{abstract}

Keywords: Diabetic nephropathy; GK rats; Type II diabetes; Renal hemodynamics; Renal disease

\section{Introduction}

Type 2 diabetes mellitus (T2DM) is a chronic progressive metabolic disease that results from impaired insulin secretion and insulin resistance leading to a dysfunction in glucose homeostasis and metabolism. Patients with T2DM experience major long-term complications related to various cardiovascular diseases such as nephropathy. Diabetic nephropathy is the leading cause of End-Stage renal disease (ESRD) in the United States [1]. Despite the introduction of several new classes of drugs for the control of diabetes, little progress has been made in developing therapies for the prevention of diabetic nephropathy. This is due in part to the lack of reliable animal models that recapitulate all the hemodynamic and histologic changes that occur in patients with diabetic nephropathy. Recently, the Animal Models of Diabetic Complications Consortium (AMDCC) defined the criteria for validating animal models of diabetic nephropathy [2]. The criteria states that the model must exhibit a $>50 \%$ decline in glomerular filtration rate (GFR) over the lifetime of the animals, a $>10$-fold increase in albuminuria and renal histologic changes including mesangial matrix expansion, nodular glomerular lesions, arteriolar hyalinosis, thickening of glomerular basement membranes, and renal interstitial fibrosis [2]. Although a large number of mouse models of diabetes have been studied by the AMDCC, the overall conclusion is that none of the existing mouse models recapitulate most of the features of human diabetic nephropathy.

The Goto-Kakizaki (GK) rat is a nonobese, normotensive model of type II diabetes [3-11].The GK strain was developed from a colony of Wistar rats by selective breeding for those rats with elevated blood glucose levels during a glucose tolerance test over many generations [12]. Diabetes in the GK rat develops between 3-4 weeks of age by impaired development of islet cells to secrete insulin in response to glucose, insulin resistance, hyperinsulinemia and abnormal glucose metabolism [13-15]. While this strain does develop some complications from diabetes, previous studies have demonstrated that GK rats are relatively resistant to the development diabetic nephropathy and do not develop progressive proteinuria, glomerulosclerosis or chronic kidney disease unless hypertension is superimposed $[3,7,8,16]$. Recently, Nobrega et al. reported on a genetically modified substrain of GK rats (T2DN) that was developed from a cross between GK and Fawn Hooded-Hypertensive (FHH) rats. These animals develop diabetes and progressive proteinuria [5].They also exhibit renal histological changes associated with diabetic nephropathy including thickening of glomerular and tubular basement membranes, glomerular hypertrophy, mesangial matrix expansion, and nodular glomerular lesions [5,17]. However, no studies to date have directly compared the time course of changes renal hemodynamics and renal injury in GK and T2DN rats or to document that T2DN rats do in fact develop chronic kidney disease (CKD). Therefore, the present study compared the temporal changes in proteinuria, renal hemodynamics and GFR in disease-resistant GK rats and the more susceptible T2DN substrain.

\section{Materials and Methods}

\section{General}

Experiments were performed on male GK and T2DN rats ranging from 3 and 18 months old. Renal function was also determined in young, nondiabetic 3 month old male Sprague Dawley (SD) rats to provide nondiabetic baseline control data. The GK and T2DN rats

*Corresponding author: Jan Michael Williams, Department of Pharmacology and Toxicology, University of Mississippi Medical Center, 2500 North State Street Jackson, MS 39216, USA, Tel: (601) 984-1634; Fax: (601) 984-1637; E-mail jmwilliams5@umc.edu

Received April 15, 2013; Accepted May 25, 2013; Published May 30, 2013

Citation: Kojima N, Slaughter TN, Paige A, Kato S, Roman RJ, et al. (2013) Comparison of the Development Diabetic Induced Renal Disease in Strains of Goto-Kakizaki Rats. J Diabetes Metab S9: 005. doi:10.4172/2155-6156.S9-005

Copyright: $\odot 2013$ Kojima N, et al. This is an open-access article distributed under the terms of the Creative Commons Attribution License, which permits unrestricted use, distribution, and reproduction in any medium, provided the original author and source are credited. 
Citation: Kojima N, Slaughter TN, Paige A, Kato S, Roman RJ, et al. (2013) Comparison of the Development Diabetic Induced Renal Disease in Strains of Goto-Kakizaki Rats. J Diabetes Metab S9: 005. doi:10.4172/2155-6156.S9-005

Page 2 of 5

were obtained from inbred colonies maintained at the University of Mississippi Medical Center. The diabetes in GK rats is the result of phase-1 deficiency in insulin secretion followed by a progressive decline in $\beta$-cell function that appears to be immune mediated $[10,11,18,19]$. The SD rats were purchased from Charles River Laboratories International, Inc. (Wilmington, MA). The rats were maintained on a standard rodent diet (Harlan Teklad 8640) and had free access to food and water throughout the study. All protocols were approved by the Animal Care Committee of the University of Mississippi Medical Center.

\section{Protocol 1: Time course of blood glucose levels and the development of proteinuria in GK and T2DN rats}

Experiments were performed on 3, 6 and 18 month old GK and T2DN rats and 3 month old SD rats that served as a nondiabetic control. The rats were placed in metabolic cages to measure protein excretion using the Bradford method (Bio-Rad Laboratories, Hercules, CA). Non-fasting blood glucose levels were measured using glucometer (Bayer HealthCare, Mishawaka, IN). At the end of the experiment, a final blood sample was drawn for measurement of plasma creatinine concentration using the Jaffe method (Wako Chemicals USA, Richmond, VA). The kidneys from GK and T2DN rats were collected, weighed and fixed in a $10 \%$ buffered formalin solution. Paraffin sections $(3 \mu \mathrm{m})$ were prepared and stained with Masson's Trichrome to assess the degree of glomerular injury, renal fibrosis and tubular necrosis (protein casts). Thirty glomeruli per section were scored in a blinded fashion on a $0-4^{+}$scale with 0 representing a normal glomerulus, $1^{+}$representing a $1-25 \%$ of loss, $2^{+}$representing a $26-50 \%$ loss, $3^{+}$ representing a $51-75 \%$ loss, and $4^{+}$representing $>75 \%$ loss of capillaries in the tuft. Additional analysis was performed to determine the degree of renal fibrosis and tubular necrosis. Images were captured using a Nikon Eclipse 55i microscope equipped with a Nikon DS-Fil color camera (Nikon Inc., Melville, NY) and analyzed for the percentage of the image stained blue for renal fibrosis and stained red for protein casts using the NIS-Elements D 3.0 software. Ten representative fields were analyzed per section.

\section{Protocol 2: Time course of the changes in renal hemodynamics in GK and T2DN rats}

Renal blood flow (RBF) and GFR were measured in separate groups of 3, 6 and 18 month-old GK and T2DN rats. The rats were anesthetized with Ketamine (30 mg/kg, i.m., Phoenix Pharmaceutical Co., St. Joseph, $\mathrm{MO})$ and Inactin (50 mg/kg, i.p., Sigma, St. Louis, MO) and placed on a warming table to maintain body temperature at $37^{\circ} \mathrm{C}$. The trachea was cannulated with PE-240 tubing to facilitate breathing and catheters were placed in the right femoral artery and vein for the measurement of mean arterial pressure (MAP) and intravenous infusions. The rats received an intravenous infusion of $0.9 \% \mathrm{NaCl}$ solution containing $2 \%$ bovine serum albumin and $2 \mathrm{mg} / \mathrm{mL}$ FITC-labeled inulin at a rate of $6 \mathrm{~mL} / \mathrm{hr}$ throughout the experiment for measurement of GFR. An ultrasound flow probe (Transonic System, Ithaca, NY) was placed on the renal artery to measure RBF. After surgery and a 30-minute equilibration period, urine and plasma samples were collected during a 30-minute collection period. Plasma and urine inulin concentrations were measured using a fluorescent microplate reader (BioTek Instruments, Winooski, VT) to determine GFR. We also performed renal hemodynamic experiments on 3 month-old SD rats to be able to determine whether there are early differences in renal hemodynamics in the GK and T2DN strains compared to a non-diabetic strain.

\section{Statistics}

Mean values \pm S.E. are presented. The significance difference in mean values between groups was determined using either an one-way or two-way analysis of variance (ANOVA) followed by a Holm-Sidak test for preplanned comparisons using the Sigma Plot 11 software (Systat Software, San Jose, CA).

\section{Results}

Time course changes in blood glucose levels and protein excretion in GK and T2DN rats

The results of these experiments are presented in Figure 1. Baseline non-fasting glucose levels averaged approximately $100 \mathrm{mg} / \mathrm{dL}$ in 3 month old SD rats. GK and T2DN rats were diabetic by 3 months of age and non-fasting blood glucose levels were elevated to the same extent $(>250 \mathrm{mg} / \mathrm{dL}$ ) in both strains (Figure 1A). Protein excretion rose from $41 \pm 2$ to $524 \pm 50 \mathrm{mg} /$ day in T2DN rats as they increased in age from 3 to 18 months of age (Figure 1B). In contrast, proteinuria only increased to $162 \pm 31 \mathrm{mg} /$ day in GK rats over this same time period.

\section{Time course changes in renal hemodynamics in GK and T2DN rats}

Temporal changes in renal hemodynamics are presented in Figure 2. MAP (measured under Inactin anesthesia) was similar and relatively stable in GK and T2DN rats as they aged from 3 to 18 months (Figure $2 \mathrm{~A})$. Baseline GFR, RBF and left kidney weight averaged $1.00 \pm 0.07$, $5.71 \pm 0.33 \mathrm{~mL} / \mathrm{min}$ and $1.20 \pm 0.05 \mathrm{~g}$, respectively, in 3 month-old nondiabetic SD rats (data not shown). RBF was markedly elevated by $45 \%$ and $146 \%$ in 3 month old T2DN rats relative to the corresponding values measured in age-matched GK and SD rats, respectively (Figure $2 \mathrm{~B})$. RBF decreased in T2DN rats and returned to values similar to that measured in GK rats at 6 and 18 months of age. GFR was elevated by $50 \%$ in 3 month old GK and T2DN rats relative to the level seen in young SD rats. GFR decreased significantly from $1.64 \pm 0.14$ to 0.72 $\pm 0.06 \mathrm{~mL} / \mathrm{min}$ in $\mathrm{T} 2 \mathrm{DN}$ rats as they increased in age from 3 to 18 months (Figure 2C). In contrast, GFR did not decrease over the same time period in GK rats. Plasma creatinine concentration was similar
A

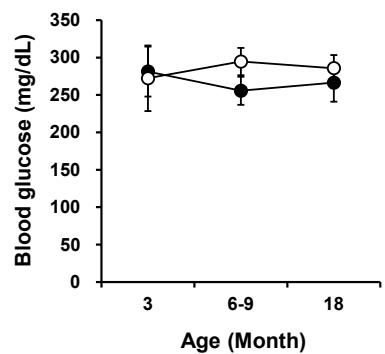

B

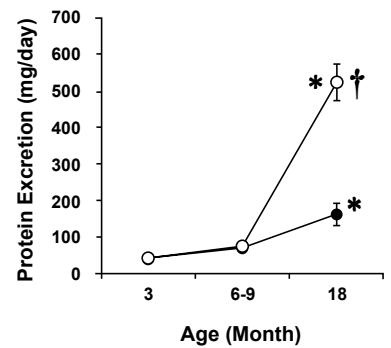

indicates a significant difference from the corresponding value at 3 months of age within the same strain and

tindicates a significant difference from the corresponding value in GK rats

Figure 1: Time course measurements of blood glucose levels (Panel $A$ ) and urinary protein excretion (Panel B) in Goto-Kakizaki (GK) rats and the modified GK substrain susceptible to diabetic nephropathy (T2DN) rats during 3 to 18 months of age. Numbers in parentheses indicate the number of rats studied per group per time period. Values are means \pm S.E. 
Citation: Kojima N, Slaughter TN, Paige A, Kato S, Roman RJ, et al. (2013) Comparison of the Development Diabetic Induced Renal Disease in Strains of Goto-Kakizaki Rats. J Diabetes Metab S9: 005. doi:10.4172/2155-6156.S9-005

in 6 month old GK and T2DN rats and averaged approximately 0.5 $\mathrm{mg} / \mathrm{dL}$. Consistent with the measured changes in GFR, we found that plasma creatinine concentration rose 4 -fold to $2.0 \mathrm{mg} / \mathrm{dL}$ over the course of the study in T2DN rats but remained relative unaltered in age matched GK rats (Figure 2D).The kidneys of 3 month old GK and T2DN rats were hypertrophied and weighed about $50 \%$ more than that seen in nondiabetic age match SD rats (SD-1.20 $\pm 0.05 \mathrm{~g}$, versus GK$1.43 \pm 0.07$ and T2DN-1.71 $\pm 0.08 \mathrm{~g}$, respectively). Over the course of study, kidney weight rose to $2.50 \pm 0.10 \mathrm{~g}$ in T2DN rats while it only increased to $1.71 \pm 0.11 \mathrm{~g}$ in GK rats.

\section{Assessment of renal injury in GK and T2DN rats}

A comparison of the typical histology of the kidneys of 18 month old GK and T2DN rats is presented in Figures 3 and 4. The kidneys from T2DN rats displayed more prominent glomerular basement membrane thickening (Figure 3E), severe focal glomerulosclerosis (Figure $3 \mathrm{E}$ ), renal interstitial fibrosis (blue staining), tubular necrosis (red staining) (Figure 3D) and arteriolar hyalinosis (Figure 3F). In contrast, kidneys of 18 month old GK rats exhibited only a minor expansion of the mesangial matrix in the glomerulus (Figure 3B) and little if any interstitial fibrosis, tubular necrosis (Figure 3A) or hyalinosis of the small arteries in the kidney (Figure $3 \mathrm{C}$ ). Glomerular injury scores were significantly elevated in 18 month old T2DN rats relative to the corresponding value in age matched GK rats (Figure 4A). The overall glomerular injury score averaged nearly 3 in T2DN rats, indicating
A

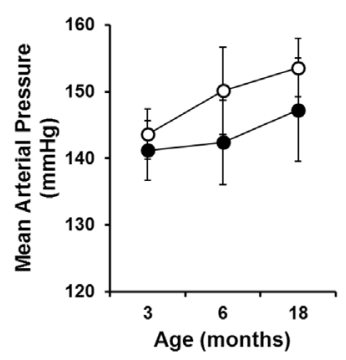

C

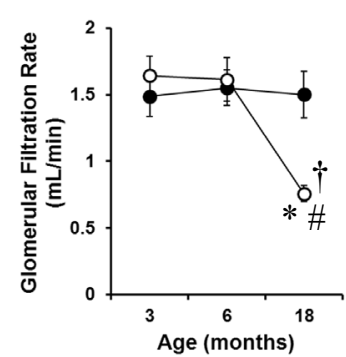

A

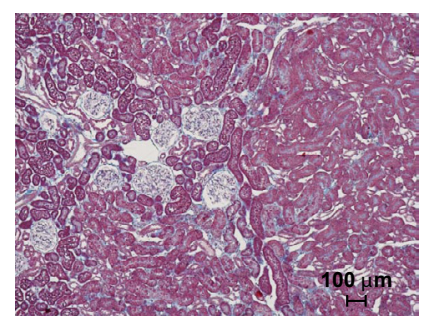

B

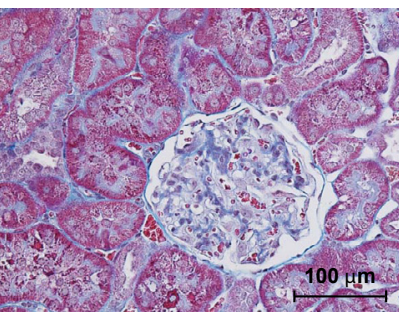

C

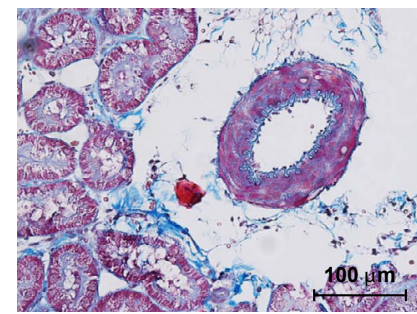

D

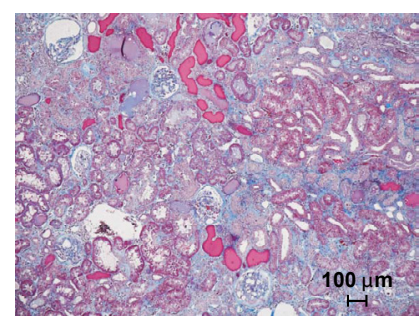

E

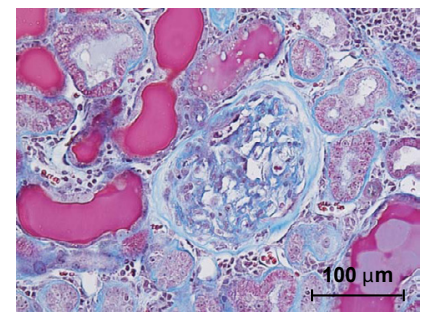

$\mathbf{F}$

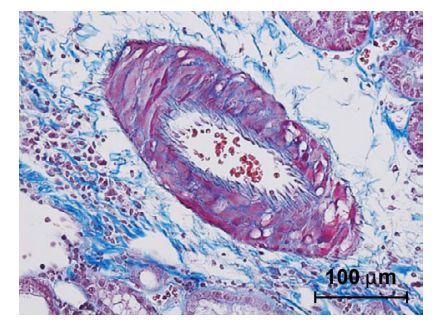

Figure 3: Representative renal histological images of therenal cortex (Panel A and $D$ ), glomeruli (Panel $B$ and $E$ ) and renal vessels (Panel $C$ and $F$ ) stained with Masson's trichrome staining in 18 month-old Goto-Kakizaki (GK) rats and the modified GK substrain susceptible to diabetic nephropathy (T2DN) rats.

that $75 \%$ of the filtration area was lost in most of the glomeruli in the kidney. Renal fibrosis (\% of blue staining) was significantly higher in T2DN rats than in GK rats (Figure 4B). Similarly, the formation of protein casts was 10 -fold higher in the 18 month old T2DN rats than in GK rats (Figure 4C).

\section{Discussion}

The GK rat is a well-accepted model of type 2 diabetes that has been widely used to study various diabetic therapies and for the study of diabetic complications [7,20]. However, previous studies have indicated that GK rats are relatively resistant to the development of diabetic nephropathy or renal end organ damage unless hypertension is superimposed $[3,16]$. Recently, the GK rat was genetically modified by crossing the GK rat with a FHH rat, a rodent model that develops chronic kidney disease [5]. The resulting T2DN strain was reported to develop diabetes by 3 months of age and develop proteinuria and histological changes in the kidney that resemble the lesions seen in patients with diabetic nephropathy including nodule formation later in life [5]. However, the time course of changes in renal hemodynamics and GFR were not measured in T2DN rats to determine to whether they develop initial hyperfiltration followed by a decline in GFR and the development of CKD. Thus, the present study compared renal hemodynamics in GK and T2DN rats as they increased in age from 3 to 18 months of age. We found that GK and T2DN rats both developed 
A

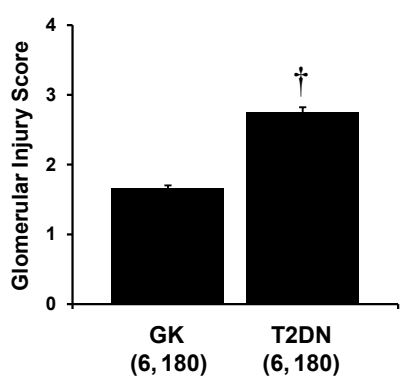

B

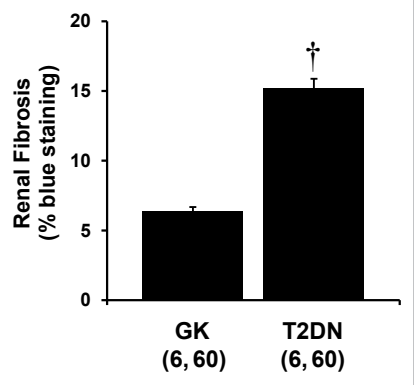

C

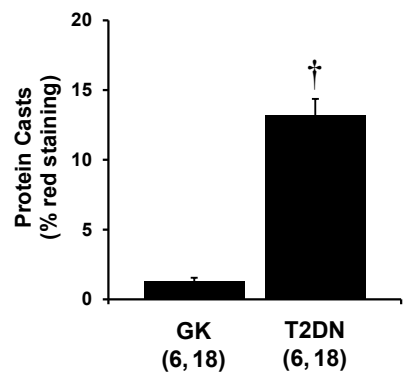

tindicates a significant difference from the corresponding value in GK rats

Figure 4: Comparison of the renal histopathology in Goto-Kakizaki (GK) rats and the modified GK substrain susceptible to diabetic nephropathy (T2DN) at 18 months of age: glomerular injury scores (Panel A), renal fibrosis (Panel B) and the formation of protein casts as an indicator of tubular necrosis (C) Numbers in parentheses indicate the number of rats and glomeruli studied per group. Values are means \pm S.E.

diabetes by 3 months of age and the degree of hyperglycemia was similar in both strains throughout the study. We observed that both strains exhibited hyperfiltration with increased RBF and GFR at 3 months of age relative to nondiabetic SD rats. However, RBF and kidney weight were higher in the T2DN strain compared to GK rats at this early time period. Nonetheless, proteinuria was similar in 3 month old GK and T2DN rats.

The T2DN rats developed severe proteinuria, renal injury and CKD over the course of the study. By 18 months of age, GFR decreased by $57 \%$ in the $\mathrm{T} 2 \mathrm{DN}$ rats whereas it remained unaltered in GK rats. Plasma creatinine concentration increased to $2.0 \mathrm{mg} / \mathrm{dL}$ in T2DN rats but remained below $1 \mathrm{mg} / \mathrm{dL}$ in GK rats. The kidneys from T2DN rats exhibited severe glomerulosclerosis, renal interstitial fibrosis and tubular necrosis, whereas the kidneys of 18 month old GK rats exhibited only minor histological changes, including thickening of renal tubular and glomerular basement membranes, an increase in glomerular size and some expansion of the mesangial matrix.

The genetic factors responsible for the increased susceptibility of the T2DN strain to develop diabetic nephropathy remains to be determined. An evaluation of the previous work by Norbrega et al. indicates that the T2DN strain was created by crossing a male $\mathrm{FHH}$ rat with a female GK rat and then repeatedly backcrossing the male progeny in each generation with female GK rats for 6 generations while selecting for animals retaining the least number of FHH alleles using a marker assisted selection [5]. Thereafter, the resulting T2DN strain was maintained by brother sister mating for more than 20 generations. A total genome scan of 543 markers across the genome revealed that only 4 markers were heterozygous in the T2DN strain for $\mathrm{FHH}$ rats indicating that $<1 \%$ of the $\mathrm{FHH}$ genome is retained on the autosomal chromosomes [5]. However, because of the breeding strategy employed the mitochondrial genome of $\mathrm{FHH}$ rats was introgressed into the T2DN strain [5].

Previous studies have indicated that FHH rats develop proteinuria and renal disease as they age and that this is associated with impairment in RBF autoregulation and elevations in glomerular capillary pressure [21-25]. Thus, we hypothesized that perhaps the increased susceptibility of T2DN rats to develop diabetic nephropathy might be due to introgression of a portion of the FHH genome that would alter renal hemodynamics in face of diabetes. Indeed, previous studies have shown that renal vasodilation and/or hyperfiltration precedes the development of proteinuria and diabetic nephropathy, whereas in later stages, diabetic patients develop chronic kidney disease and hypertension [26]. In the current study, we found that RBF was significantly higher in the T2DN rats than relative to GK rats. This finding is consistent with the view that glomerular capillary pressure may be elevated in T2DN rats than GK rats early and this might trigger the development of renal disease. However, GFR and the degree of hyperfiltration were similar in T2DN and GK rats, suggesting that differences in renal hemodynamics do not contribute to the increased susceptibility of T2DN rats to develop diabetic nephropathy. The more likely conclusion probably lies in the differences in the mitochondrial genome between T2DN and GK rats that may dictate differences in renal metabolic function and the generation of reactive oxygen species between the strains in response to the diabetic state.

The AMDCC recently defined the criteria for validating rodent models of human diabetic nephropathy [2]. The AMDCC defined diabetic nephropathy as a $50 \%$ decline in GFR over the lifetime of the animals, a 10-fold increase in albuminuria compared with controls for that strain at the same age, and the kidneys must exhibit renal histopathological lesions similar to patients with diabetic nephropathy [2]. The current study demonstrated that the T2DN strain exhibits all the criteria established by the AMDCC for a rodent model of diabetic nephropathy (Table 1). In this regard, we confirmed that GFR decreases by $\geq 50 \%$ in T2DN rats over a 15 month period and they develop CKD as evidenced by a 4 -fold elevation in plasma creatinine concentration. Similar to previous work by Nobrega et al. [5], we found that proteinuria increased $\geq 10$-fold in the present study in T2DN rats as they increased in age from 3 to 18 months of age. Moreover, the kidneys of T2DN rats display all of the histologic features of diabetic nephropathy including thickening of the basement membrane, mesangial matrix expansion, severe glomerulosclerosis, renal vascular hyalinosis, renal interstitial fibrosis and tubular necrosis. Moreover, Nobrega et al. reported that

Table 1: Criteria for diabetic nephropathy in GK and T2DN rats.

\begin{tabular}{|l|c|c|}
\hline Criteria & GK & T2DN \\
\hline Early increase in GFR (hyperfiltration) & ++ & ++ \\
\hline$>10$-fold increase in albuminuria & + & +++ \\
\hline Mesangial matrix expansion & + & +++ \\
\hline Thickening of glomerular basement membrane & + & +++ \\
\hline Renal interstitial fibrosis & + & +++ \\
\hline Arteriolar hyalinosis & NO & YES \\
\hline Nodular glomerular lesions & NO & YES \\
\hline $50 \%$ decline in renal function & NO & YES \\
\hline
\end{tabular}

These criteria were set forth by the AMDCC for a rodent model of diabetic nephropathy

'indicates the severity within the individual criteria as compared to nondiabetic rat strains 
Citation: Kojima N, Slaughter TN, Paige A, Kato S, Roman RJ, et al. (2013) Comparison of the Development Diabetic Induced Renal Disease in Strains of Goto-Kakizaki Rats. J Diabetes Metab S9: 005. doi:10.4172/2155-6156.S9-005

Page 5 of 5

they also exhibit nodular glomerular lesions that are characteristic of human diabetic nephropathy $[5,17]$. Taken together, these data support the view that the T2DN rat is a genetic rodent model of type II diabetes that is well suited for testing the effectiveness of new therapeutic approaches to slow the progression of diabetic nephropathy.

\section{Acknowledgements}

This work was supported in part by a NIH grant HL36279 to R. Roman and an American Heart Association Scientist Development Grant and a PhRMA Foundation Starter Grant awarded to J. Williams. This work was also supported in part from a research contract from Taisho Pharmaceutical Co., Ltd.

\section{References}

1. Collins AJ, Foley RN, Herzog C, Chavers BM, Gilbertson D, et al. (2010) Excerpts from the US Renal Data System 2009 Annual Data Report. Am J Kidney Dis 55: S1-420, A6-7.

2. Brosius FC 3rd, Alpers CE, Bottinger EP, Breyer MD, Coffman TM, et al. (2009) Mouse models of diabetic nephropathy. J Am Soc Nephrol 20: 2503-2512.

3. Janssen U, Riley SG, Vassiliadou A, Floege J, Phillips AO (2003) Hypertension superimposed on type II diabetes in Goto Kakizaki rats induces progressive nephropathy. Kidney Int 63: 2162-2170.

4. Janssen U, Vassiliadou A, Riley SG, Phillips AO, Floege J (2004) The quest for a model of type II diabetes with nephropathy: the Goto Kakizaki rat. J Nephrol 17: 769-773.

5. Nobrega MA, Fleming S, Roman RJ, Shiozawa M, Schlick N, et al. (2004) Initial characterization of a rat model of diabetic nephropathy. Diabetes 53: 735-742.

6. Olearczyk JJ, Quigley JE, Mitchell BC, Yamamoto T, Kim IH, et al. (2009) Administration of a substituted adamantyl urea inhibitor of soluble epoxide hydrolase protects the kidney from damage in hypertensive Goto-Kakizaki rats. Clin Sci (Lond) 116: 61-70.

7. Phillips AO, Baboolal K, Riley S, Gröne H, Janssen U, et al. (2001) Association of prolonged hyperglycemia with glomerular hypertrophy and renal basement membrane thickening in the Goto Kakizaki model of non-insulin-dependent diabetes mellitus. Am J Kidney Dis 37: 400-410.

8. Riley SG, Steadman R, Williams JD, Floege J, Phillips AO (1999) Augmentation of kidney injury by basic fibroblast growth factor or platelet-derived growth factor does not induce progressive diabetic nephropathy in the Goto Kakizak model of non-insulin-dependent diabetes. J Lab Clin Med 134: 304-312.

9. Sato N, Komatsu K, Kurumatani $\mathrm{H}$ (2003) Late onset of diabetic nephropathy in spontaneously diabetic GK rats. Am J Nephrol 23: 334-342.

10. Akash MS, Rehman K, Sun H, Chen S (2013) Sustained delivery of IL-1Ra from PF127-gel reduces hyperglycemia in diabetic GK-rats. PLoS One 8: e55925.

11. Akash MS, Rehman K, Sun H, Chen S (2013) Interleukin-1 receptor antagonist improves normoglycemia and insulin sensitivity in diabetic Goto-Kakizaki-rats. Eur J Pharmacol 701: 87-95.
12. Goto $Y$, Suzuki K, Ono T, Sasaki M, Toyota T (1988) Development of diabetes in the non-obese NIDDM rat (GK rat). Adv Exp Med Biol 246: 29-31.

13. Movassat J, Saulnier C, Serradas P, Portha B (1997) Impaired development of pancreatic beta-cell mass is a primary event during the progression to diabetes in the GK rat. Diabetologia 40: 916-925.

14. Ostenson CG, Khan A, Abdel-Halim SM, Guenifi A, Suzuki K, et al. (1993) Abnormal insulin secretion and glucose metabolism in pancreatic islets from the spontaneously diabetic GK rat. Diabetologia 36: 3-8.

15. Picarel-Blanchot F, Berthelier C, Bailbé D, Portha B (1996) Impaired insulin secretion and excessive hepatic glucose production are both early events in the diabetic GK rat. Am J Physiol 271: E755-762.

16. Cheng ZJ, Vaskonen T, Tikkanen I, Nurminen K, Ruskoaho H, et al. (2001) Endothelial dysfunction and salt-sensitive hypertension in spontaneously diabetic Goto-Kakizaki rats. Hypertension 37: 433-439.

17. Williams JM, Zhang J, North P, Lacy S, Yakes M, et al. (2011) Evaluation of metalloprotease inhibitors on hypertension and diabetic nephropathy. Am J Physiol Renal Physiol 300: F983-998.

18. Akash MS, Rehman K, Chen S (2013) Role of inflammatory mechanisms in pathogenesis of type 2 diabetes mellitus. J Cell Biochem 114: 525-531.

19. Kawai J, Ohara-Imaizumi M, Nakamichi Y, Okamura T, Akimoto Y, et al. (2008) Insulin exocytosis in Goto-Kakizaki rat beta-cells subjected to long-term glinide or sulfonylurea treatment. Biochem J 412: 93-101.

20. Yagihashi S, Goto Y, Kakizaki M, Kaseda N (1978) Thickening of glomerula basement membrane in spontaneously diabetic rats. Diabetologia 15: 309-312.

21. Burke M, Pabbidi M, Fan F, Ge Y, Liu R, et al. (2013) Genetic basis of the impaired renal myogenic response in FHH rats. Am J Physiol Renal Physio 304: F565-577.

22. López B, Ryan RP, Moreno C, Sarkis A, Lazar J, et al. (2006) Identification of a QTL on chromosome 1 for impaired autoregulation of RBF in fawn-hooded hypertensive rats. Am J Physiol Renal Physiol 290: F1213-1221.

23. Van Dokkum RP, Alonso-Galicia M, Provoost AP, Jacob HJ, Roman RJ (1999) Impaired autoregulation of renal blood flow in the fawn-hooded rat. Am J Physiol 276: R189-196.

24. van Dokkum RP, Sun CW, Provoost AP, Jacob HJ, Roman RJ (1999) Altered renal hemodynamics and impaired myogenic responses in the fawn-hooded rat. Am J Physiol 276: R855-863.

25. Williams JM, Burke M, Lazar J, Jacob HJ, Roman RJ (2011) Tempora characterization of the development of renal injury in $\mathrm{FHH}$ rats and $\mathrm{FHH} .1 \mathrm{BN}$ congenic strains. Am J Physiol Renal Physiol 300: F330-338.

26. Zelmanovitz T, Gerchman F, Balthazar AP, Thomazelli FC, Matos JD, et al (2009) Diabetic nephropathy. Diabetol Metab Syndr 1: 10. 